\title{
Mini-Mental State Examination: Avaliação dos Novos Dados Normativos no Rastreio e Diagnóstico do Défice Cognitivo
}

\section{Mini-Mental State Examination: Screening and Diagnosis of Cognitive Decline, Using New Normative Data}

\author{
Isabel SANTANA $\triangle^{1,2,3}$, Diana DURO ${ }^{2,3}$, Raquel LEMOS ${ }^{4,5}$, Vanessa COSTA ${ }^{4}$, Miguel PEREIRA ${ }^{3}$, Mário R. SIMÕES $4,6,7$, \\ Sandra FREITAS $1,4,6,7$ \\ Acta Med Port 2016 Apr;29(4):240-248 - http://dx.doi.org/10.20344/amp.6889
}

\section{RESUMO}

Introdução: O Mini-Mental State Examination é o teste de rastreio de défice cognitivo/demência mais difundido. No nosso país têm-se utilizado pontuações de corte definidas por grupos de literacia, mas existem novas propostas sustentadas por estudos mais representativos. Propomo-nos confirmar a influência da idade e da escolaridade no desempenho, avaliar a capacidade discriminativa dos novos dados normativos e testar a acuidade diagnóstica das pontuações de corte validadas para o défice cognitivo ligeiro e para as formas mais prevalentes de demência.

Material e Métodos: O estudo incluiu 1441 participantes escolarizados, divididos em sete subgrupos: Défice cognitivo ligeiro, doença de Alzheimer, demência fronto-temporal, demência vascular, demência com corpos de Lewy, controlo-comunidade e controlo-clínicamemória.

Resultados: Em conjunto, idade e escolaridade explicam 10,4\% da variância dos resultados no Mini-Mental State Examination, com ambas contribuindo significativamente para a predição dos resultados. A acuidade diagnóstica com base nos dados normativos mais recentes foi sempre superior à conseguida com as pontuações de corte de validação, revelando uma especificidade excelente (superior a 90\%) e uma sensibilidade também excelente para a doença de Alzheimer ligeira (91\%), boa para demência com corpos de Lewy (78\%), baixa para o défice cognitivo ligeiro (65\%) e demência fronto-temporal e demência vascular (55\%).

Discussão e Conclusões: O desempenho no Mini-Mental State Examination é influenciado pela idade e pela escolaridade, apoiando a utilização de dados normativos que considerem estas variáveis. Com esta abordagem, o Mini-Mental State Examination poderá ser um instrumento sensível e específico para o rastreio da doença de Alzheimer em todos os níveis de cuidados de saúde, mas a acuidade de diagnóstico é limitada noutras situações frequentes em consultas especializadas, como o défice cognitivo ligeiro ou outras formas de demência.

Palavras-chave: Cognição; Défice Cognitivo Ligeiro; Demência; Doença de Alzheimer; Testes Neuropsicólogicos.

\section{ABSTRACT}

Introduction: The Mini-Mental State Examination is the most commonly used cognitive screening test. In Portugal, the cut-off scores are defined according to literacy groups, but different proposals have been recommended by more representative studies. We therefore propose to confirm the influence of demographical variables, such as age and education, in the subject's performance; evaluating the discriminant ability of the new normative data; and to further examine the diagnostic acuity of the validated cut-off scoring for mild cognitive impairment and for the most prevalent types of dementia.

Material and Methods: Our study includes 1441 educated subjects, divided into seven subgroups: Mild cognitive impairment, Alzheimer's disease, frontotemporal dementia, vascular dementia, dementia with Lewy bodies, community-controls and memory cliniccontrols.

Results: Altogether age and education explain $10.4 \%$ of the Mini-Mental State Examination results variance, with both variables contributing significantly to the results' prediction. The diagnostic acuity based on the most recent normative data was always higher than the one obtained through the validation cut-off scoring, revealing an overall excellent specificity (superior to $90 \%$ ) and different sensitivity values: excellent for mild Alzheimer's disease (91\%), good for dementia with Lewy Bodies (78\%) and low for mild cognitive impairment (65\%), frontotemporal dementia and vascular dementia (55\%).

Discussion and Conclusions: The performance on the Mini-Mental State Examination is influenced by age and education, supporting the use of normative data that consider those variables. With this approach, the Mini-Mental State Examination could be a sensitive and specific instrument for the Alzheimer's disease screening among all healthcare levels. Nevertheless, its diagnostic acuity is limited in other conditions frequently seen in memory clinics, such as Mild Cognitive Impairment and other types of dementia. Keywords: Alzheimer Disease; Cognition; Dementia; Mild Cognitive Impairment; Neuropsychological Tests.

\footnotetext{
1. Centro de Neurociências e Biologia Celular. Universidade de Coimbra. Coimbra. Portugal.

2. Faculdade de Medicina. Universidade de Coimbra. Coimbra. Portugal.

3. Consulta de Demência, Serviço de Neurologia. Centro Hospitalar e Universitário de Coimbra. Coimbra. Portugal.

4. Faculdade de Psicologia e de Ciências da Educação. Universidade de Coimbra. Coimbra. Portugal.

5. Instituto Biomédico de Investigação da Luz e Imagem. Universidade de Coimbra. Coimbra. Portugal.

6. Centro de Investigação do Núcleo de Estudos e Intervenção Cognitivo Comportamental. Universidade de Coimbra. Coimbra. Portugal.

7. Laboratório de Avaliação Psicológica. Faculdade de Psicologia e de Ciências da Educação. Universidade de Coimbra. Coimbra. Portugal.

$\bowtie$ Autor correspondente: Isabel Santana. isabeljsantana@gmail.com

Recebido: 07 de agosto de 2015 - Aceite: 30 de novembro de 2015 | Copyright @ Ordem dos Médicos 2016
} 


\section{INTRODUÇÃO}

O Mini-Mental State Examination (MMSE) ${ }^{1}$ foi desenvolvido na década de 70 com o propósito de identificar indivíduos com deterioração cognitiva em estudos de campo. ${ }^{2}$ Actualmente é o teste cognitivo breve mais difundido e com a maior diversidade de aplicações no âmbito da avaliação do estado mental, nomeadamente em estudos epidemiológicos, de investigação clínica e na prática clínica. Neste último enquadramento, é utilizado em todos os níveis de cuidados de saúde, quer como instrumento de rastreio do défice cognitivo e demência, quer na avaliação longitudinal e da resposta ao tratamento. ${ }^{2,3}$ Originalmente, foi proposto o valor 23/24 como cut-off universal para deterioração cognitiva/demência. ${ }^{4}$ Este valor de corte continua a ser utilizado em muitos países, apesar de se reconhecer um efeito de penalização nos mais idosos e com baixo nível de literacia, verificando-se um efeito contrário (de teto) em indivíduos com alta escolaridade. ${ }^{5-7}$ Existe um consenso na literatura indicando que o desempenho nesta prova é muito influenciado por diversas variáveis demográficas, sobressaindo sistematicamente a escolaridade e, nalguns estudos, a idade..$^{8-10}$

Em Portugal, os primeiros estudos de adaptação transcultural, normalização e validação foram realizados por Guerreiro e colaboradores na década de $90^{11,12}$ numa amostra de conveniência essencialmente urbana, confirmando-se a importância da escolaridade no desempenho global. De acordo com este critério, definiram-se valores de corte diferenciados de acordo com a literacia ( 15 pontos em indivíduos analfabetos; 22 para 1 - 11 anos; 27 para > 11 anos). Em 2009, Morgado et al ${ }^{13}$ atualizaram os dados normativos para o nosso país. Basearam-se numa amostra comunitária robusta de indivíduos com idades acima dos 50 anos, recolhida na área metropolitana de Lisboa. Neste estudo, a literacia identificou-se novamente como a principal variável preditiva da pontuação total do teste, propondo-se os valores de corte ( $5^{\circ}$ percentil) de 22 para 0 - 2 anos de literacia, 24 para 3 - 6 anos e 27 para literacia $\geq$ sete anos. Recentemente, Freitas et al ${ }^{14}$ desenvolveram um estudo semelhante (comunitário/amostra de conveniência), mas com um espectro etário mais largo (idade superior aos 25 anos) e com uma amostra que se pode considerar representativa da população continental portuguesa (amostragem estratificada considerando as principais variáveis sociodemográficas e com uma distribuição semelhante ao universo populacional do nosso país). ${ }^{14}$ Analisaram um número significativo de variáveis demográficas e de saúde e concluíram que a idade e a escolaridade eram ambas significativas, sendo, em conjunto, responsáveis por $26 \%$ da variância das pontuações do MMSE. De acordo com estes resultados, propuseram dados normativos considerando essas duas variáveis (Anexo 1 - http://www.actamedicaportuguesa.com/revista/ index.php/amp/editor/downloadFile/6889/19736). Paralelamente, o mesmo grupo desenvolveu estudos de validação do MMSE num centro terciário, abrangendo os principais grupos nosológicos de défice cognitivo e demência: défice cognitivo ligeiro (DCL) e doença de Alzheimer (DA), ${ }^{15}$ de- mência frontotemporal $(\mathrm{DFT})^{16}$, demência vascular (DV) ${ }^{17}$ e demência com corpos de Lewy (DCLewy). ${ }^{18} \mathrm{~A}$ pontuação de corte mais favorável para o DCL foi o valor de 29 , correspondendo-lhe valores de sensibilidade $67 \%$, de especificidade $72 \%$ e acuidade diagnóstica de $69 \% \cdot{ }^{15}$ Curiosamente, na demência identificou-se consistentemente o cut-off de 26 , embora com valores de acuidade de diagnóstico diferenciados nos diversos grupos patológicos e com resultados mais favoráveis para a DA,,$^{19}$ tal como apresentado no Anexo 2 (http://www.actamedicaportuguesa.com/revista/ index.php/amp/editor/downloadFile/6889/19737).

A reavaliação das novas propostas de aferição/classificação noutras populações é essencial, ${ }^{20}$ sendo igualmente indispensável promover análises periódicas das pontuações de corte, uma vez que a evolução cultural das populações pode modificar a sua acuidade diagnóstica. ${ }^{21}$ No caso particular do MMSE, o estudo de Morgado et $\mathrm{a}^{22}$ é elucidativo dessa evolução: num intervalo de 20 anos houve uma mudança nos critérios normativos, com valores de corte superiores para cada grupo de literacia. Este efeito foi muito expressivamente denominado pelos autores como "efeito de época".

No sentido de contribuir para a referida avaliação sistemática e dinâmica do MMSE, definimos como objetivos deste estudo:

1) reavaliar a influência da idade e da escolaridade no desempenho e na capacidade discriminativa do teste;

2) avaliar especificamente a validade da última proposta de dados normativos baseados nessas duas variáveis (Freitas et al) $)^{14}$;

3) testar a acuidade diagnóstica das pontuações de corte propostas para a população portuguesa nos estudos de validação para DCL e para as formas mais prevalentes de demência, ${ }^{15-18}$ utilizando novos coortes patológicos mais robustos;

4) partindo desses resultados, propomo-nos analisar o contributo e as limitações do MMSE no diagnóstico hierárquico do défice cognitivo nos diversos níveis de cuidados de saúde, desde o screening básico de "declínio cognitivo" até ao diagnóstico nosológico mais específico.

\section{MATERIAL E Métodos \\ Desenho do estudo}

A amostra total compreende duas amostras de conveniência de doentes e controlos, escolarizados, divididos em sete subgrupos: (i) DCL; (ii) DA; (iii) DFT; (iv) DV; (v) DCLewy; (vi) Controlo-Comunidade e (vii) Controlo-ClínicaMemória. Os doentes foram recrutados sistematicamente na Consulta de Demência do Centro Hospitalar e Universitário de Coimbra (CHUC) e numa Clínica de Memória da mesma cidade (CM); o grupo Controlo-Comunidade reúne grupos normativos de diferentes áreas geográficas, recolhidos no âmbito de estudos de aferição de vários instrumentos (excluindo o estudo de aferição do MMSE) tendo como locais de recrutamento os cuidados de saúde primários (Centros de Saúde, Unidades de Saúde Familiar) e Centros 
de Dia; o grupo Controlos-Clínica-Memória é constituído por sujeitos com desempenho normal para a idade e para escolaridade na avaliação neuropsicológica aprofundada, identificados na base da referida CM.

\section{Selecção dos Participantes}

1. Grupos patológicos - O protocolo de avaliação dos doentes, tanto na CM como na Consulta de Demência, inclui a história clínica e o exame neurológico, bem como uma bateria abrangente de caracterização neuropsicológica, funcional e psicológica, que engloba os instrumentos listados no Anexo 3 (http://www.actamedicaportuguesa.com/ revista/index.php/amp/editor/downloadFile/6889/19738). De salientar que o instrumento em estudo (MMSE) não foi considerado como orientação ou critério de diagnóstico. A investigação laboratorial e de imagem implementada segue as normas nacionais e internacionais para excluir demências reversíveis. Com base nesta investigação, é proposto um diagnóstico clínico: DCL amnésico mono ou multidomínios $^{23}$ ou demência. ${ }^{24}$ Neste estudo, a inclusão foi restringida a doentes com DCL e Demência Ligeira, utilizando-se como critério de classificação a pontuação global da escala Clinical Dementia Rating - CDR (Hughes et al, 1982; Garrett et al, 2008; Santana et al, 2015) (estádio 0,5/DCL e $1 /$ demência ligeira). A classificação nas principais formas de demência baseia-se nos critérios internacionais para os diagnósticos prováveis de: $\mathrm{DA}^{25}$; $\mathrm{DFT}^{26}$; DCLewy ${ }^{27}$; DV. ${ }^{28}$ Finalmente, foram considerados critérios de exclusão: (i) demências de gravidade moderada/severa (CDR $\geq 2$ ); (ii) doentes portadores de défices sensoriais comprometendo a avaliação cognitiva; (iii) indivíduos com outras situações médicas ou neurológicas relevantes em termos de repercussão cognitiva ou instáveis; (iv) os doentes com classificação duvidosa ou patologia mista.

2. Grupos de Controlo - O grupo Controlo-Comunidade inclui sujeitos recrutados em vários projetos de investigação do nosso Grupo desenvolvidos na Região Centro e no Alentejo e que têm em comum os seguintes critérios de inclusão: (i) português como língua nativa e escolaridade realizada em Portugal; (ii) ausência de défices motores, visuais e auditivos que pudessem influenciar o desempenho nos testes; (iii) desempenho "normal" nas provas integradas na bateria de avaliação do estudo (ver em baixo); (iv) autonomia nas atividades de vida diária; (v) ausência de história de alcoolismo, abuso de substâncias ou medicação com possível impacto negativo na cognição (p. ex., benzodiazepinas de longa duração ou em doses elevadas, antidepressivos tricíclicos e neurolépticos); (vi) ausência de patologia de natureza neurológica, psiquiátrica ou médica/ sistémica com potencial repercussão cognitiva; (vii) sem sintomatologia depressiva significativa, como especificado em baixo.

A informação subjacente a estes critérios de inclusão é obtida numa entrevista estruturada, que abrange dados sociodemográficos, um inventário do estado clínico actual, antecedentes clínicos pessoais e hábitos anteriores. Os participantes admitidos com base neste inquérito passam à segunda fase de seleção que visa comprovar que são cognitivamente saudáveis (os instrumentos estão elencados no Anexo 3 - http://www.actamedicaportuguesa.com/ revista/index.php/amp/editor/downloadFile/6889/19738). Consideram-se elegíveis sujeitos classificados na CDR como cognitiva e funcionalmente normais (pontuação global na CDR $=0$ ) e sem depressão grave (pontuação $\leq 20$ ) de acordo com a Escala de Depressão Geriátrica - GDS 30 (Yesavage et al, 1983; Barreto et al, 2008).

O Grupo Controlo-Clínica-Memória é constituído por sujeitos maioritariamente com queixas subjectivas de atenção/memória, nos quais a avaliação neuropsicológica aprofundada se revelou normal (Anexo 3). Foram excluídos deste grupo os sujeitos com depressão grave (pontuação > 20) de acordo com a GDS - 30.

\section{Instrumento de estudo}

O MMSE é um teste clássico de papel/lápis ( 1 folha), de aplicação fácil e rápida (5 a 10 minutos), sendo o tempo de execução não cronometrado. Na avaliação de rotina (e bem assim neste estudo que pretende avaliar as características do instrumento na sua aplicação quotidiana) utilizamos a versão adaptada à população portuguesa, assim como as instruções de aplicação e as regras de pontuação propostas por Guerreiro. ${ }^{29} \mathrm{O}$ teste é constituído por 30 questões (pontuadas com valor 0 - quando o indivíduo não responde ou dá uma resposta incorrecta ou 1 - quando o examinado fornece uma resposta correcta) organizadas em seis domínios cognitivos: Orientação - 5 itens de orientação temporal e 5 de orientação espacial; Retenção - repetição de 3 palavras ('Pêra, Gato, Bola'); Atenção e Cálculo - série de 5 subtrações de 3 iniciando-se no número trinta; Evocação - o examinando tem de evocar espontaneamente as três palavras retidas; Linguagem - inclui dois itens de Nomeação (Lápis e Relógio), um de Repetição de uma frase ('O rato roeu a rolha'), um de Compreensão de ordem verbal com três passos (Pegar numa folha com a mão direita, dobrar ao meio e colocar num local a designar), um de Compreensão de ordem escrita ('Feche os olhos'), um de Escrita espontânea - uma frase com sentido e gramaticalmente correta; Capacidade Construtiva (cópia de dois pentágonos intersectados por dois ângulos). O teste permite obter uma pontuação máxima de 30 pontos, sendo que as pontuações mais elevadas indicam melhores desempenhos.

\section{Análise Estatística}

A análise estatística foi realizada com recurso ao programa Statistical Package for the Social Sciences (versão 20, IBM SPSS, Chicago, IL). Para a caracterização da amostra foi utilizada a estatística descritiva e para a comparação entre os grupos o teste $t$-student para duas amostras independentes. A comparação entre grupos realizou-se através de ANOVA e testes Post-hoc. O coeficiente de correlação de Pearson e a regressão linear múltipla (método Enter) permitiram investigar a relação dos resultados obtidos no MMSE com as variáveis sociodemográficas. Para a análise 
da precisão diagnóstica foi calculada a sensibilidade (a probabilidade de indivíduos com comprometimento cognitivo terem um teste positivo) e a especificidade (a probabilidade de indivíduos sem comprometimento cognitivo terem um teste negativo).

\section{RESULTADOS}

A amostra do estudo é composta por 1441 participantes, $60 \%(n=864)$ do género feminino, cuja idade média é de $69,75 \pm 9,83$ [Min. = 36, Máx. = 96] anos e a escolaridade média de 6,90 \pm 4,50 [Min. = 1, Máx. = 18] anos. A amostra total compreende sete subgrupos: (i) DCL ( $n=500)$; (ii) DA ( $n=250) ;($ iii) DFT ( $n=112)$; (iv) DV ( $n=130)$; (v) DCLewy ( $n=59$ ); (vi) Controlo-Comunidade ( $n=318$ ) e (vii) Controlo-Clínica-Memória $(\mathrm{n}=72)$. Os dados relativos à caracterização sociodemográfica e desempenho no MMSE destes subgrupos são apresentados na Tabela 1.

A comparação entre grupos revelou diferenças estatisticamente significativas ao nível da idade $\left(F_{(6,1434)}=28,468\right.$, $p<0,001)$, verificando-se, de acordo com os testes Post-Hoc, que o subgrupo Controlo-Clínica-Memória seria o mais jovem e os subgrupos clínicos DA e DCLewy apresentavam idades mais avançadas, sem diferirem significativamente entre si. Os restantes subgrupos não revelaram diferenças estatisticamente significativas entre si, mas diferiram significativamente do subgrupo mais jovem e destes subgrupos com mais idade.

Ao nível da literacia, foram igualmente observadas diferenças estatisticamente significativas entre os grupos $\left(F_{(6,1434)}=11,110, p<0,001\right)$, embora, de acordo com os testes Post-hoc, apenas o subgrupo Controlo-Clinica-Memória apresentou uma escolaridade média significativamente superior à escolaridade média dos restantes subgrupos, que não diferiram entre si.

A comparação entre grupos ao nível do desempenho no MMSE evidenciou um padrão mais heterogéneo de diferenças nas pontuações totais médias obtidas $\left(F_{(6,1429)}=\right.$ $218,471, p<0,001)$. O pior desempenho médio verificou- -se na DA, seguindo-se os pacientes com DCLewy. Estes subgrupos diferiam significativamente entre si e comparativamente aos restantes subgrupos. Os pacientes com DFT e DV revelaram desempenhos similares, significativamente superiores aos subgrupos DA e DCLewy e significativamente inferiores aos restantes subgrupos. O subgrupo $\mathrm{DCL}$ obteve pontuações médias significativamente inferiores aos subgrupos controlo e significativamente superiores a todos os outros subgrupos clínicos. Não foram observadas diferenças significativas entre os desempenhos dos dois subgrupos controlo.

Os resultados obtidos no MMSE apresentaram uma correlação positiva significativa com a escolaridade $(r=$ $0,24, p=0,01)$ e uma correlação negativa significativa com a idade $(r=0,25, p=0,01)$. Os resultados da regressão linear múltipla (método Enter) revelaram que ambas as variáveis contribuem significativamente para a predição dos resultados no MMSE $\left(F_{(2,1433)}=83,086, p<0,001\right.$; Idade: $\beta$ $=-0,220, t=-8,696, p<0,001$; Escolaridade: $\beta=0,202, t=$ $7,984, p<0,001)$. Em conjunto, idade e escolaridade explicam $10,4 \%$ da variância dos resultados no MMSE, sendo que idades mais avançadas e níveis de escolaridade mais baixos aumentam a probabilidade de piores desempenhos.

Para a análise da sensibilidade e especificidade dos dados normativos recentemente propostos para a população portuguesa, ${ }^{14}$ considerou-se o critério de 1,5 desvios padrão abaixo do valor proposto nas normas para idade/escolaridade, apresentadas no Anexo 1 (http://www.actamedicaportuguesa.com/revista/index.php/amp/editor/downloadFile/6889/19736). Os resultados são apresentados, por subgrupos, na Tabela 2. A mesma análise foi realizada considerando os pontos de corte propostos pelos estudos de validação para a população portuguesa com DCL, ${ }^{15} \mathrm{DA},{ }^{15}$ DFT, ${ }^{16} \mathrm{DV}^{17}$ e DCLewy, ${ }^{18}$ ou seja, examinando os pontos de corte apresentados no Anexo 2 (http://www.actamedicaportuguesa.com/revista/index.php/amp/editor/downloadFile/6889/19737): (i) $\geq 29$ pontos para ausência de declínio cognitivo clinicamente significativo, (ii) $<29$ pontos para o

Tabela 1 - Caracterização dos subgrupos

\begin{tabular}{|c|c|c|c|c|c|}
\hline Grupo & $\mathbf{n}$ & $\begin{array}{c}\text { Género } \mathbf{F} \\
\%(n)\end{array}$ & Idade & Escolaridade & MMSE \\
\hline DCL & 500 & $65,2 \%(326)$ & $\begin{array}{c}69,05 \pm 9,04 \\
{[43-91]}\end{array}$ & $\begin{array}{c}7,23 \pm 4,64 \\
{[1-18]}\end{array}$ & $\begin{array}{c}27,19 \pm 2,31 \\
{[19-30]}\end{array}$ \\
\hline DA & 250 & $58,8 \%(147)$ & $\begin{array}{c}74,14 \pm 7,69 \\
{[51-91]}\end{array}$ & $\begin{array}{c}6,07 \pm 4,04 \\
{[1-17]}\end{array}$ & $\begin{array}{c}21,63 \pm 3,38 \\
{[15-29]}\end{array}$ \\
\hline DFT & 112 & $47,3 \%(53)$ & $\begin{array}{c}67,53 \pm 8,73 \\
{[43-87]}\end{array}$ & $\begin{array}{c}6,66 \pm 4,04 \\
{[1-17]}\end{array}$ & $\begin{array}{c}25,19 \pm 3,78 \\
{[16-30]}\end{array}$ \\
\hline DV & 130 & $43,1 \%(56)$ & $\begin{array}{c}68,98 \pm 9,91 \\
{[45-89]}\end{array}$ & $\begin{array}{c}5,86 \pm 3,74 \\
{[1-17]}\end{array}$ & $\begin{array}{c}25,35 \pm 3,32 \\
{[18-30]}\end{array}$ \\
\hline DCLewy & 59 & $64,4 \%(38)$ & $\begin{array}{c}76,10 \pm 6,47 \\
{[61-89]}\end{array}$ & $\begin{array}{c}6,88 \pm 4,78 \\
{[2-17]}\end{array}$ & $\begin{array}{c}23,22 \pm 3,76 \\
{[16-30]}\end{array}$ \\
\hline Controlo-Comunidade & 318 & $62,9 \%(200)$ & $\begin{array}{c}69,50 \pm 10,84 \\
{[40-96]}\end{array}$ & $\begin{array}{c}6,74 \pm 4,60 \\
{[1-18]}\end{array}$ & $\begin{array}{c}28,81 \pm 1,31 \\
{[23-30]}\end{array}$ \\
\hline Controlo-Clinica-Memória & 72 & $61,1 \%(44)$ & $\begin{array}{c}60,10 \pm 10,62 \\
{[36-82]}\end{array}$ & $\begin{array}{c}10,46 \pm 4,41 \\
{[4-16]}\end{array}$ & $\begin{array}{c}29,01 \pm 1,34 \\
{[22-30]}\end{array}$ \\
\hline
\end{tabular}

O género é apresentado em percentagem de participantes do género feminino e respetiva frequência. As variáveis Idade, Escolaridade e MMSE são apresentadas em média \pm desvio padrão e respetiva amplitude [mínimo-máximo]. 
Tabela 2 - Análise da sensibilidade e especificidade dos pontos de corte normativos no MMSE

\begin{tabular}{|c|c|c|c|}
\hline \multirow{2}{*}{ Grupo } & \multirow{2}{*}{$\mathbf{n}$} & \multicolumn{2}{|c|}{ Estudo normativo (Freitas et al, 2015) } \\
\hline & & Sensibilidade & Especificidade \\
\hline DCL & 500 & $64,6 \%$ & -- \\
\hline DA & 250 & $91,2 \%$ & -- \\
\hline DFT & 112 & $54,5 \%$ & -- \\
\hline DV & 130 & $54,6 \%$ & -- \\
\hline DCLewy & 59 & $78,0 \%$ & -- \\
\hline Controlo-Comunidade & 318 & -- & $96,5 \%$ \\
\hline Controlo-Clinica-Memória & 72 & -- & $91,7 \%$ \\
\hline
\end{tabular}

Tabela 3 - Análise da sensibilidade e especificidade dos pontos de corte dos estudos de validação clínica no MMSE

\begin{tabular}{lccc}
\hline Grupo & $\mathbf{n}$ & \multicolumn{2}{c}{ Estudos de validação clínica } \\
\hline DCL & & Sensibilidade & Especificidade \\
DA & 500 & $31,8 \%$ & -- \\
DFT & 250 & $84,4 \%$ & -- \\
DV & 112 & $44,6 \%$ & -- \\
DCLewy & 130 & $44,7 \%$ & -- \\
Controlo-Comunidade & 59 & $67,8 \%$ & -- \\
Controlo-Clinica-Memória & 318 & -- & $68,9 \%$ \\
\hline
\end{tabular}

Tabela 4 - Distribuição por intervalos de pontuações, de acordo com os pontos de corte dos estudos de validação clínica do MMSE na população portuguesa

\begin{tabular}{lccc}
\hline MMSE & $\begin{array}{c}\text { Controlos } \\
(n=390)\end{array}$ & $\begin{array}{c}\text { DCL } \\
(\mathrm{n}=498)\end{array}$ & $\begin{array}{c}\text { Demência } \\
(\mathrm{n}=548)\end{array}$ \\
\hline$[29-30]$ & $274(70,3 \%)$ & $175(35,0 \%)$ & $56(10,2 \%)$ \\
{$[26-28]$} & $106(27,2 \%)$ & $214(43,0 \%)$ & $128(23,4 \%)$ \\
$<26$ & $10(2,5 \%)$ & $109(22,0 \%)$ & $364(66,4 \%)$ \\
\hline
\end{tabular}

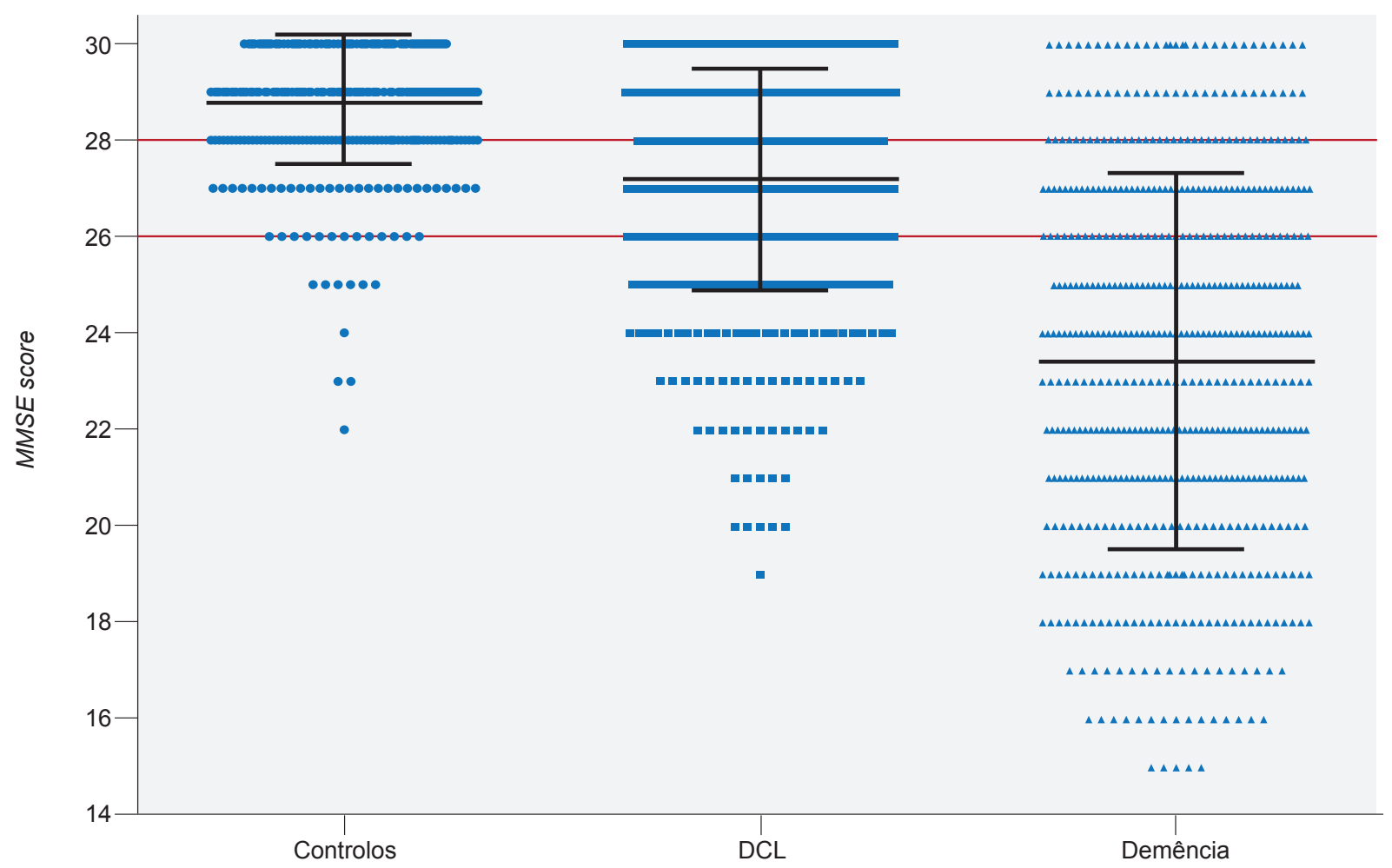

Figura 1 - Distribuição dos grupos clínicos 
DCL e (iii) < 26 pontos para presença de declínio em pacientes com demência devida a DA, DFT, DV ou DCLewy (resultados apresentados na Tabela 3).

Visámos analisar a capacidade do MMSE como auxiliar na discriminação entre as três grandes categorias nosológicas - normal, DCL e Grupo Demência (reunindo DA, DFT, DV e DCLewy): os resultados da distribuição dos participantes dos três grupos pelos intervalos de pontuações sugeridas pelos pontos de corte dos estudos de validação clínica na população portuguesa (Anexo 2 - http://www. actamedicaportuguesa.com/revista/index.php/amp/editor/ downloadFile/6889/19737) são apresentados na Tabela 4 e representados na Fig. 1.

\section{DISCUSSÃO}

Com o envelhecimento progressivo das populações, ${ }^{30,31}$ o declínio cognitivo e as demências tornaram-se parte das principais patologias do nosso tempo e uma prioridade assistencial. ${ }^{32}$ Neste contexto, o MMSE como teste de rastreio de demência adquiriu uma grande notoriedade, sendo referenciado em praticamente todas as normas de orientação diagnóstica internacionais ${ }^{33-36}$ e em Portugal, ${ }^{37}$ bem como nos principais critérios de diagnóstico..$^{27,28,38}$ No entanto, o MMSE foi criado na década de 70 e, neste quase meio século de existência, surgiu uma nova categoria nosológica - "defeito cognitivo ligeiro" - aceite atualmente como fase pré-demencial. ${ }^{39,40} \mathrm{O}$ foco no declínio ligeiro exige a utilização de instrumentos de rastreio sensíveis e, neste novo enquadramento, são apontadas limitações ao MMSE: uma reduzida complexidade das tarefas de memória/ linguagem conduzindo a uma relativa insensibilidade para o DCL e a ocorrência de falsos negativos nos indivíduos com escolaridade elevada; a ausência de tarefas para a avaliação das funções executivas, o que compromete a sua sensibilidade na identificação de patologias frequentes, como a DFT ou DV. 16,41-45 Paradoxalmente, estudos longitudinais de DCL/ DA-prodrómica demonstram que o MMSE contribui para prever o declínio progressivo e a evolução para demência quando associado a marcadores biológicos como a ressonância magnética ${ }^{46}$ ou os biomarcadores do LCR. ${ }^{47}$ Além disso, este instrumento continua a ser utilizado como critério praticamente universal de gravidade ou estadiamento, para inclusão de doentes em ensaios clínicos. ${ }^{48}$ Em Portugal, a actualidade deste estudo justifica-se pela proposta de pontuações de corte mais exigentes, de acordo com os estudos de Morgado et $\mathrm{al}^{13}$ e de Freitas et al. ${ }^{14} \mathrm{De}$ salientar que neste último estudo os autores já não incluíram iliteratos, uma opção que também se baseou na sua reduzida representatividade na sociedade actual. Em consequência, e dado que esta nossa análise de validação se refere especificamente a essas pontuações de corte, também não foram estudados analfabetos, pelo que os resultados apresentados não serão aplicáveis a pessoas sem escolaridade formal. No entanto, excluindo os analfabetos, procurámos obter amostras de sujeitos cognitivamente normais e de doentes que fossem representativas da realidade assistencial do nosso país, desde a comunidade até as consultas específicas de memória. Assim, o grupo controlo da comunidade representa os utentes que se apresentam aos médicos de família para caraterização cognitiva ou avaliação diagnóstica, enquanto o segundo grupo controlo é constituído pelos utentes das consultas especializadas de memória que levantam as mesmas questões diagnósticas aos neurologistas. A maioria referia queixas subjetivas de atenção/memória mas a avaliação neuropsicológica aprofundada, com testes aferidos para a idade e para escolaridade, revelou um desempenho normal, pelo que podemos considerá-los como controlos cognitivos neste enquadramento específico de validação de testes de rastreio. $\mathrm{Na}$ verdade, em relação às pontuações médias no MMSE não se observaram diferenças significativas na comparação com o grupo comunitário, apesar das diferenças de proveniência, da idade e da escolaridade não terem sido controladas na análise dos desempenhos. No que se refere aos grupos patológicos do nosso estudo, a distribuição reflete a proporcionalidade habitual de um centro terciário, com preponderância dos défices cognitivos mais ligeiros (DCL), os quais, de acordo com as normas vigentes, têm indicação para avaliação em clínicas especializadas. .33,34 Também nos grupos de demência estudados, as características sociodemográficas (idade e género) e a proporcionalidade são congruentes com os estudos de prevalência, estando mais representada a DA, seguida da DV e da DFT e por fim a DC Lewy. Em termos de desempenho no MMSE, o subgrupo DCL obteve pontuações médias significativamente inferiores aos subgrupos controlo e significativamente superiores a todos os outros subgrupos clínicos de demência, o que indica que o MMSE consegue diferenciar estas três grandes categorias nosológicas (controlos-DCL-demência). Entre os diferentes subgrupos de demência os piores desempenhos foram para DA, seguida da DC Lewy e uma pontuação média superior e equivalente para a DFT e a DV. Dado que o MMSE não foi considerado no estadiamento de gravidade podemos concluir que esta gradação de desempenhos é uma primeira evidência de que o MMSE tem um constructo (memória, linguagem e capacidade construtiva) mais vocacionado para detetar os défices típicos da DA e da DC Lewy, sendo menos sensível a situações caracterizadas por disfunção frontal como a DFT e a DV. ${ }^{14,49-51}$

No que respeita à influência das variáveis sociodemográficas no desempenho global do teste, confirmámos que a idade e a escolaridade contribuíram ambas para a predição dos resultados e, saliente-se, com um peso semeIhante, conforme indicado pelo valor beta. O valor obtido de 10,4 , tem menor magnitude que o apontado para outras provas de rastreio, nomeadamente para o MoCA, cuja predição era $49 \%$ para o conjunto destas variáveis, com um peso de $42 \%$ para a escolaridade e $7 \%$ para a Idade. ${ }^{52} \mathrm{~A}$ confirmação de que a Idade é uma variável significativa e importante nos resultados obtidos com o MMSE reforça a adequação dos últimos resultados normativos de Freitas et al, ${ }^{14}$ cujas pontuações de corte são ponderadas com base na escolaridade e na idade. A apreciação na especialidade desses scores normativos (representada na Tabela 2) 
sublinha a excelente especificidade da prova (valores superiores a $90 \%$ ), ou seja, tem capacidade para identificar não afetados e não gera muitos falsos positivos. ${ }^{33}$ Relativamente à sensibilidade, confirmam-se globalmente as tendências evidenciadas noutros estudos, ou seja:

i) uma sensibilidade excelente para a DA ligeira (91\%), sendo este valor até mais favorável do que tem sido reportado por alguns estudos ${ }^{53}$;

ii) para a DC Lewy também uma moderada a boa sensibilidade $(78 \%)$, semelhante à sugerida no estudo de Ala et $\mathrm{al}^{54}$;

iii) uma sensibilidade agrupada baixa $(64,6 \%)$ para o $D C L$, levando-nos a concordar que o teste é pouco adequado para o rastreio do declínio cognitivo mais ligeiro ${ }^{3,15}$;

iv) valores baixos de sensibilidade para a DFT e a DV $(55 \%)$, indicando também a desadequação do teste ao rastreio destas patologias, provavelmente porque a disfunção frontal, dominante, não é avaliada pelo MMSE. ${ }^{16,41-45}$

Definimos também como objetivo testar a acuidade diagnóstica das pontuações de corte propostas no estudo de validação para o DCL e para as formas mais prevalentes de demência. Comparando os resultados atuais de sensibilidade e de especificidade (referidos na Tabela 3), com os originalmente encontrados (Anexo 2 - http://www. actamedicaportuguesa.com/revista/index.php/amp/editor/ downloadFile/6889/19737), verifica-se um decréscimo global, tanto em termos de sensibilidade como de especificidade, com exceção da sensibilidade para DA cujos valores são semelhantes (respetivamente $84 \%$ e $85 \%$ ). A comparação dos resultados baseados em dados normativos com os resultantes dos estudos de validação (comparação entre os resultados apresentados respetivamente nas Tabelas 2 e 3) apresenta exatamente a mesma tendência, com uma redução drástica da especificidade de excelente para boa/ razoável, e uma perda de sensibilidade de cerca de $7 \%$ para a DA, de cerca de $50 \%$ para o DCL (baixou de $65 \%$ para $32 \%$ ) e de pelo menos $10 \%$ para os restantes grupos de demência. A Tabela 4 e a Fig. 1 permitem-nos identificar melhor o tipo de erros de diagnóstico que surgem com a utilização de dados de validação, podendo concluir-se que no grupo controlo há cerca de $30 \%$ de falsos positivos, sendo que a maioria dos casos seria classificada como DCL; no grupo de demência há cerca de $34 \%$ de falsos negativos, também com a grande maioria a classificar erroneamente na categoria DCL. O DCL é o grupo mais penalizado, com $57 \%$ de erros, maioritariamente falsos negativos. Devemos assim concluir que a classificação individual baseada exclusivamente em dados de validação sem atender à idade e escolaridade do indivíduo é muito falível, sendo preferível, no que respeita ao MMSE, utilizar os dados normativos estabelecidos de acordo com estas variáveis.

Tendo em conta informação colhida neste trabalho é possível propor uma abordagem ou protocolo de avaliação em que a avaliação com o MMSE pode ser complementada com outros instrumentos de rastreio ou provas neuropsicológicas mais específicas, operacionalizados de acordo com os níveis de exigência de diagnóstico e especialização dos serviços. Assim, a nível dos cuidados primários, propomos o MMSE como o instrumento de primeira linha, dada a facilidade e rapidez de aplicação e o conhecimento já bastante difundido do teste entre os clínicos que lidam com situações geriátricas. Considerando a demonstrada especificidade do MMSE mediante a utilização de dados normativos, os utentes com classificação de défice cognitivo poderão ser propostos com bastante segurança como portadores de défice cognitivo/demência e referenciados para consultas específicas de memória com vista a um diagnóstico nosológico e tratamento mais específicos. Esta hipótese diagnóstica será ainda mais sustentada se a pontuação no MMSE for inferior a 26, o score sugestivo de demência, conforme indicado pelos estudos de validação. Nos casos considerados normais de acordo com a base normativa, será muito improvável que sofram de doença de Alzheimer e poderá igualmente excluir-se com bastante segurança o diagnóstico de DC Lewy, uma vez que a sensibilidade do MMSE para estas duas situações é boa. No entanto, como a sua acuidade de diagnóstico é bastante mais limitada para o DCL, a DFT e a DV, aconselhamos que este grupo "normal" seja avaliado complementarmente por um instrumento de rastreio mais sensível. A escolha deste instrumento deverá basear-se em estudos que permitam selecionar o que demonstre melhor capacidade discriminativa. No contexto de consultas de especialidade onde a prevalência de DCL e de outras formas de demência não-Alzheimer é elevada, o MMSE deverá ser preterido como instrumento de rastreio, em favor de outro mais sensível e com um constructo mais abrangente. Mesmo assim, o MMSE terá que manter-se como teste de rastreio de primeira linha nos iliteratos e poderá, complementarmente, ser utilizado como instrumento de estadiamento de gravidade, bem como na avaliação longitudinal ao longo de todo o curso da doença.

Este estudo tem limitações que queremos salientar. Em primeiro lugar e como foi já referido, a nossa análise não incluiu analfabetos pelo que este estudo não acrescenta informação sobre este grupo de literacia. Os estádios moderados-severos de demência, também não foram objeto de análise, uma vez que a acuidade diagnóstica e utilidade do MMSE nesses estádios está bem documentada na literatura. ${ }^{15,55,56}$ É importante ainda sublinhar que não foi objeto deste estudo comparar a acuidade de diagnóstico das diversas propostas normativas do MMSE e que as considerações expostas se aplicam ao contexto cultural específico de Portugal, não podendo ser extrapoladas para outras realidades sociais e culturais.

\section{CONCLUSÃO}

O nosso estudo comprova que o desempenho no MMSE é muito influenciado pela idade e pela escolaridade, apoiando a utilização de dados normativos que considerem ambas as variáveis. Com este tipo de abordagem, demonstrou-se que o MMSE é um instrumento sensível e específico para o rastreio de DA e DC Lewy. Em contraponto, a sua acuidade de diagnóstico é limitada noutras situações 
importantes e frequentes em contexto assistencial como o DCL, a DFT e a DV. Consideramos ainda que esta análise, baseada numa segunda amostragem, reforça a adequação da proposta normativa e de pontos de corte de Freitas et al $^{14}$ e permite ilustrar o contributo e as limitações do MMSE no diagnóstico hierárquico do défice cognitivo nos diversos níveis de cuidados de saúde.

\section{PROTECÇÃO DE PESSOAS E ANIMAIS}

Os autores declaram que os procedimentos seguidos estavam de acordo com os regulamentos estabelecidos pelos responsáveis da Comissão de Investigação Clínica

\section{REFERÊNCIAS}

1. Folstein M, Folstein S, McHugh P. Mini-Mental State: A practical method for grading the cognitive state of patients for the clinician. J Psychiatr Res. 1975;12:189-98.

2. Nieuwenhuis-Mark RE. The death knoll for the MMSE: Has it outlived its purpose?. J Geriatr Psychiatry Neurol. 2010;23:151-7.

3. Mitchell AJ. A meta-analysis of the accuracy of the mini-mental state examination in the detection of dementia and mild cognitive impairment. J Psychiatr Res. 2009;43:411-31.

4. O' Connor DW, Pollitt PA, Hyde JB, Fellows JL, Miller ND, Brook CP, et al. The reliability and validity of the Mini-Mental State in a British community survey. J Psychiatr Res. 1989;23:87-96.

5. Gallacher JE, Eliood PC, Hopkinson C, Rabbitt PM, Stollery BT, Sweetnam PM, et al. Cognitive function in the Caerphilly study: Associations with age, social class, education and mood. Eur J Epidemiol. 1999;15:161-9.

6. Han C, Jo SA, Jo I, Kim E, Park MH, Kang Y. An adaptation of the Korean Mini-Mental State Examination (K-MMSE) in elderly Koreans: Demographic influence and population-based norms (the AGE study). Arch Gerontol Geriatr. 2008;47:302-10.

7. Moraes C, Pinto JA, Lopes MA, Litvoc J, Bottino CM. Impact of sociodemographic and health variables on Mini-Mental State Examination in a community-based sample of older people. Eur Arch Psychiatry Clin Neurosci. 2010;260:535-42.

8. Anderson TM, Sachdev PS, Brodaty H, Trollor J, Andrews G. Effects of sociodemographic and health variables on Mini-Mental State Exam scores in older Australians. Am J Geriatr Psychiatry. 2007;15:467-76.

9. Bravo G, Hébert R. Age and education specific reference values for the Mini-Mental and Modified Mini-Mental State Examination derived from a non-demented elderly population. Int J Geriatr Psychiatry. 1997;12:1008-18.

10. Matallana D, Santacruz C, Cano C, Reyes P, Sampaer-Ternent R, Markides KS, et al. The relationship between educational level and MiniMental State Examination domains among older Mexican Americans. J Geriatr Psychiatry Neurol. 2011;24:9-18.

11. Guerreiro M, Silva AP, Botelho M, Leitão O, Castro-Caldas A, Garcia C. Adaptação à população portuguesa da tradução do Mini Mental State Examination. Rev Port Neurol. 1994;1,9.

12. Guerreiro M. Contributo da Neuropsicologia para o estudo das demências [Tese de Doutoramento]. Lisboa: Universidade de Lisboa; 1998.

13. Morgado J, Rocha CS, Maruta C, Guerreiro M, Martins IP. Novos valores normativos do Mini-Mental State Examination. Sinapse. 2009;2:10-6.

14. Freitas S, Simões MR, Alves L, Santana I. Mini Mental State Examination (MMSE): Normative study for the Portuguese population in a community stratified sample. Appl Neuropsych Adults. 2015;22:311-9.

15. Freitas S, Simões MR, Alves L, Santana I. Montreal Cognitive Assessment (MoCA): Validation study for Mild Cognitive Impairment and Alzheimer's Disease. Alzheimer Dis Assoc Disord. 2013;27:37-43.

16. Freitas S, Simões MR, Alves L, Duro D, Santana I. Montreal Cognitive Assessment (MoCA): Validation study for Frontotemporal Dementia. J Geriatr Psychiatry Neurol. 2012;25:146-54.

17. Freitas S, Simões MR, Alves L, Vicente M, Santana I. Montreal Cognitive Assessment (MoCA): Validation study for Vascular Dementia. e Ética e de acordo com a Declaração de Helsínquia da Associação Médica Mundial.

\section{CONFIDENCIALIDADE DOS DADOS}

Os autores declaram ter seguido os protocolos do seu centro de trabalho acerca da publicação de dados.

\section{CONFLITOS DE INTERESSE}

Os autores declaram não terem qualquer conflito de interesse relativamente ao presente artigo.

\section{FONTES DE FINANCIAMENTO}

Os autores declaram não ter recebido subsídios ou bolsas para a elaboração do artigo.

J Int Neuropsychol Soc. 2012;18:1031-40.

18. Costa V, Freitas S, Simões MR, Santana I. Estudo exploratório de validação do Montreal Cognitive Assessment (MoCA) na Demência com Corpos de Lewy. Sinapse;15:228.

19. Freitas S, Simões MR, Alves L, Santana I. Mini Mental State Examination (MMSE). In: Mário R. Simões, Isabel Santana \& Grupo de Estudos de Envelhecimento Cerebral e Demência (GEECD), editors. Escalas e Testes na demência. $3^{\text {rd }}$ ed. Lisboa: Novartis; 2015;p.18-23.

20. Larner AJ. Introduction to cognitive screening instruments: Rationale, desiderata, and assessment of utility. In: Mitchell AJ, editor. Cognitive screening instruments. London: Springer-Verlag; 2013;p.1-14.

21. Mitchell A J. The Mini-Mental State Examination (MMSE): An update on its diagnostic validity for cognitive disorders. In: Mitchell AJ, editor. Cognitive screening instruments. London: Springer-Verlag; 2013,p.1546.

22. Morgado J, Rocha CS, Maruta C, Guerreiro M, Mártins IP. Cut-off scores in MMSE: A moving target?. Eur J Neurol. 2010;17:692-5.

23. Albert MS, DeKosky ST, Dickson D, Dubois B, Feldman HH, et al. The diagnosis of mild cognitive impairment due to Alzheimer's disease: Recommendations from the National Institute on Aging-Alzheimer's Association workgroups on diagnostic guidelines for Alzheimer's disease. Alzheimers Dement. 2011;7:270-9.

24. American Psychiatric Association. DSM-IV-TR: Diagnostic and statistical manual of mental disorders. $4^{\mathrm{a}}$ ed. Lisboa: Climepsi Editors; 2002.

25. McKhann GM, Knopman DS, Chertkow H, Hyman BT, Jack CR, et al.. The diagnosis of dementia due to Alzheimer's disease: Recommendations from the National Institute on Aging-Alzheimer's Association workgroups on diagnostic 38 guidelines for Alzheimer's disease. Alzheimers Dement. 2011;7:263-9.

26. Neary D, Snowden JS, Gustafson L, Passant U, Stuss D, Black S Frontotemporal lobar degeneration: A consensus on clinical diagnostic criteria. Neurology. 1998;51:1546-54.

27. McKeith IG, Dickson DW, Lowe J, Emre M, O'Brien JT, Feldman H, et al. Diagnosis and management of dementia with Lewy bodies: third report of the DLB consortium. Neurology. 2005;65:1863-72.

28. Román GC, Tatemichi TK, Erkinjuntti T, Cummings JL, Masdeu JC, Garcia JH, et al. Vascular dementia: Diagnostic criteria for research studies. Report of the NINDS-AIREN International Workshop. Neurology. 1993;43:250-60.

29. Guerreiro M, Fonseca S, Barreto J, Garcia C. Escala de avaliação da Doença de Alzheimer. In: Grupo de Estudos de Envelhecimento Cerebral e Demências, editor. Escalas e testes na demência. $2^{\text {nd }}$ ed. Lisboa: GEECD; 2008. p.41-58.

30. Federal Interagency Forum on Aging-Related Statistics. Older americans 2010: Key indicators of well-being. [consultado 2015 Jun 10]. Dísponível em: http:// http://www.agingstats.gov/.

31. European Commission. The social situation in the European Union 2009. [consultado 2015 Jun 10]. Disponível em: http://www.ec.europa. eu/eurostat.

32. Santana I, Farinha F, Freitas S, Rodrigues V, Carvalho A. Epidemiologia da demência e da Doença de Alzheimer em Portugal: Estimativas da prevalência e dos encargos financeiros com a medicação. Acta Med Port. 2015,28:182-8. 
33. National Institute for Health and Clinical Excellence. Dementia: supporting people with dementia and their carers in health and social care. National Clinical Practice Guideline Number 42. London: National Institute for Health and Clinical Excellence; 2011.

34. Hort J, O'Brien JT, Gainotti G, Pirttila T, Popescu BO, Rektorova I, et al. EFNS guidelines for the diagnosis and management of Alzheimer's disease. Eur J Neurol. 2010;17:1236-48.

35. Sorbi S, Hort J, Erkinjuntti T, Fladby T, Gainotti G, Gurvit H, et al. Guidelines on the diagnosis and management of disorders associated with dementia. Eur J Neurol. 2012;19:1159-79.

36. California Workgroup on Guidelines for Alzheimer's Disease Management. Guidelines for Alzheimer's Disease Management. State of California: Department of Public Health. 2008.

37. Direcção Geral da Saúde. Norma 053/2011 de 27/12/2011. Abordagem Diagnóstica e Terapêutica das Alterações Cognitivas (Demências; Doença de Alzheimer). [consultado 2015 Jun 10]. Disponível em: https:// www.dgs.pt/.

38. McKhann G, Drachman D, Folstein M, Katzman R, Price D, Stadlan EM, et al. Clinical diagnosis of Alzheimer's disease: Report of the NINCDSADRDA Work Group under the auspices of Department of Health and Human Services Task Force on Alzheimer's disease. Neurology. 1984;4:939-44.

39. Dubois B., Feldman HH, Jacova C, Dekosky ST, Barberger-Gateau P, Cummings J, Delacourte A, Galasko D, Gauthier S, Jicha G, Meguro K, O'brien J, Pasquier F, Robert P, Rossor M, Salloway S, Stern Y, Visser PJ, Scheltens P. Research criteria for the diagnosis of Alzheimer"s disease: revising the NINCDS-ADRDA criteria. Lancet Neurol. 2007; 6: 734-46.

40. Dubois B, Feldman HH, Jacova C, Hampel H, Molinuevo JL, Blennow K, et al. Advancing research diagnostic criteria for Alzheimer's disease: the IWG-2 criteria. Lancet Neurol. 2014;13:614-29.

41. Hutchinson AD, Mathias JL. Neuropsychological deficits in frontotemporal dementia and Alzheimer's disease: a meta-analytic review. J Neurol Neurosur Psychiatry. 2007;78:917-28.

42. Ihl R, Frölich TD, Martin, EM, Maurer K. Differential validity of psychometric tests in dementia of Alzheimer type. Psychiatry Res. 1992;44:93-106.

43. Markwick A, Zamboni G, Jager C. Profile of cognitive subtest impairment in the Montreal Cognitive Assessment (MoCA) in a research cohort with normal Mini-Mental State Examination (MMSE) scores. J Clin Exp Neuropsych. 2012;34:750-7.

44. Naugle RI, Kawczak K. Limitations of the Mini-Mental State Examination.
Cleve Clin J Med. 1989;56:277-81.

45. Tombaugh TN, Mclntyre NJ. The Mini-Mental State Examination: A comprehensive review. J Am Geriatr Soc. 1992;40:922-35.

46. Kovacevic S, Rafii MS, Brewer JB, Alzheimer's Disease Neuroimaging Initiative. High-throughput, fully automated volumetry for prediction of MMSE and CDR decline in mild cognitive impairment. Alzheimer Dis Assoc Disord. 2009;23:139-45.

47. Hall A, Muñoz-Ruiz M, Mattila J, Koikkalainen J, Tsolaki M, Mecocci P, et al. Generalizability of the disease state index prediction model for identifying patients progressing from mild cognitiveimpairment to Alzheimer's disease. J Alzheimers Dis. 2015;44:79-92.

48. Vellas B, Andrieu S, Sampaio C, Coley N, Wilcock G; European Task Force Group. Endpoints for trials in Alzheimer's disease: a European task force consensus. Lancet Neurol. 2008;7:436-50.

49. Gregory CA, Orrell M, Sahakian B, Hodges JR. Can frontotemporal dementia and Alzheimer's disease be differentiated using a brief battery of tests? Int J Geriatr Psychiatry. 1997;12:375-83.

50. Hodges JR, Patterson K, Ward R, Garrard P, Bak T, Perry R, et al. The differentiation of semantic dementia and frontal lobe dementia (temporal and frontal variants of frontotemporal dementia) from Alzheimer's disease: A comparative neuropsychological study. Neuropsychology. 1999;13:31-40.

51. Mathuranath PS, Nestor PJ, Berrios GE, Rakowicz W, Hodges JR. A brief cognitive test battery to differentiate Alzheimer's disease and frontotemporal dementia. Neurology. 2000;55:1613-20.

52. Freitas S, Simões MR, Alves L, Santana I. Montreal Cognitive Assessment (MoCA): Influence of sociodemographic and health variables. Arch Clin Neuropsych. 2012;27:165-75.

53. Boustani M, Peterson B, Hanson L, Harris R, Lohr K. Screening for dementia in primary care: a summary of the evidence for the U.S. Preventive Services Task Force. Ann Intern Med. 2003;138:927-37.

54. Ala TA, Hughes LF, Kyrouac GA, Ghobrial MW, Elble RJ. The MiniMental State exam may help in the differentiation of dementia with Lewy bodies and Alzheimer's disease. Int J Geriatr Psychiatry. 2002;17:5039.

55. Harvan JR, Cotter V. An evaluation of dementia screening in the primary care setting. J Am Acad Nurse Pract. 2006;18:351-60

56. O'Bryant SE, Humphreys JD, Smith GE, Ivnik RJ, Graff-Radford NR, Petersen RC, et al. Detecting dementia with the Mini-Mental State Examination (MMSE) in highly educated individuals. Arch Neurol. 2008;65:963-7. 